\title{
ASV-Abrechnung
}

\section{Ausschlüsse im EBM gelten nicht immer}

\author{
Die Mehrfachberechnung von ASV-Leistungen nach EBM sind jetzt \\ möglich. Den vollen Punktwert gibt es für mehrmals abgerechnete \\ Leistungen allerdings nicht.
}

m Zusammenhang mit der ambulanten spezialfachärztlichen Versorgung (ASV) kommt es häufig vor, dass mehrere, auch fachgruppengleiche Ärzte in die Versorgung eines Patienten einbezogen sind. Das führt zu Mehrfachabrechnung von Leistungen der ASV, die laut EBM (einheitlicher Bewertungsmaßstab) eigentlich nur einmal im Behandlungsfall berechnungsfähig sind. Bisher wurden diese Leistungen nicht mehrfach vergütet - und genau das hat der Erweiterte Bewertungsausschuss kürzlich geändert.

Seit Ende 2015 gilt die neue Regelung, nach der fachgruppengleiche Ärzte in der ASV in einem Behandlungsfall solche Leistungen der ASV auch mehrfach abrechnen können. Es werden alle Leistungen vergütet, wenn Patienten in einem Quartal mehrere Ärzte derselben Fachgruppe eines ASV-Teams aufsuchen. Allerdings wurde für diesen Fall eine Abschlagsregelung beschlossen. Diese Abschlagsregelung beruht auf der Tatsache, dass in der ASV die Behandlung in einem interdisziplinären Team im Vordergrund steht und Doppeluntersuchungen und -behandlungen vermieden werden sollen.

\section{Fachgruppenfall geschaffen}

Um diese Mehrfachabrechnung zu ermöglichen, musste im EBM auch eine Anpassung der Definition des Behandlungsfalles durchgeführt werden. Dies vor allem deshalb, weil an der ASV auch Krankenhäuser teilnehmen. Die Definition des Behandlungsfalles gemäß 3.1 der Allgemeinen Bestimmungen des EBM bezieht sich jedoch auf die Behandlung durch dieselbe Arztpraxis. Vor diesem Hintergrund war eine Definition des Behandlungsfalles für die ASV notwendig geworden.

Neu eingeführt wurde dazu der Fachgruppenfall. Der Fachgruppenfall gilt als Behandlungsfall für Krankenhäuser sowie Vertragsärzte in Berufsausübungsgemeinschaften (BAG) und Medizinischen Versorgungszentren (MVZ). Sind dort mehrere Ärzte einer Fachgruppe im selben ASV-Kernteam tätig, kann immer nur einer die Leistung je Patient im Quartal abrechnen.

Für Vertragsärzte in Einzelpraxen sieht der Beschluss vor, dass der Behandlungsfall als Arztfall definiert wird. Er umfasst die Behandlung desselben Versicherten durch denselben Arzt in einem

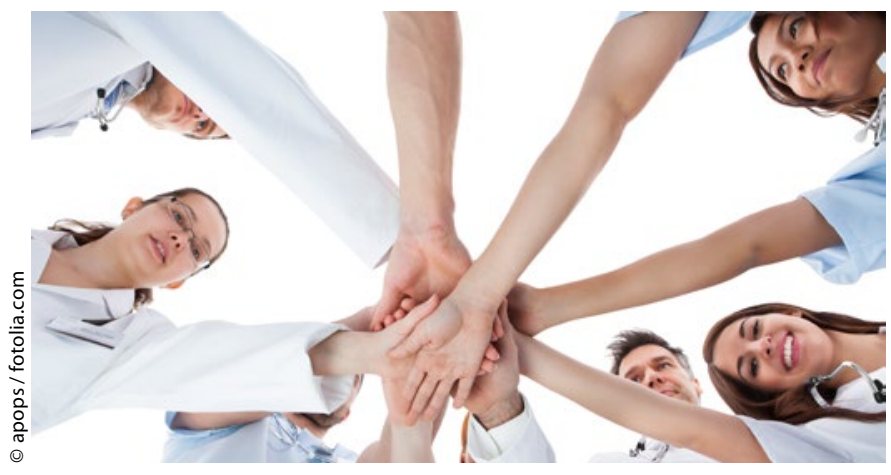

Gemeinsam stark: Bei der Abrechnung von Leistungen bieten sich ASV-Teams seit 2015 neue Chancen.
Kalendervierteljahr. Für die Abrechnung heißt das, dass die Ärzte alle ASVLeistungen ihres jeweiligen Fachgebietes abrechnen können. Allerdings gilt hier die Abschlagsregelung, wenn mehr als ein ASV-Kernteam-Mitglied derselben Fachgruppe dieselbe behandlungsfalldefinierte Leistung für einen Patienten abrechnet.

Für Ihre Abrechnung ergeben sich daraus primär keine Änderungen. Als ASV-berechtigte Ärzte rechnen Sie Ihre ASV-Leistungen wie gewohnt ab. Die Krankenkassen haben dann die Aufgabe zu prüfen, ob ASV-Leistungen mehrfach durchgeführt und abgerechnet wurden. Die Abschlagsregelung kommt nämlich zur Anwendung, wenn in einem ASV-Kernteam Ärzte derselben Fachgruppe dieselbe behandlungsfalldefinierte Gebührenordnungsposition in einem Behandlungsfall mehrmals abrechnen. Dabei spielt es keine Rolle, ob die Ärzte eines ASV-Kernteams in einer Einzelpraxis, einer Berufsausübungsgemeinschaft (BAG), einem medizinischen Versorgungszentrum (MVZ) oder im Krankenhaus arbeiten.

\section{Abschlag von zehn oder 15 Prozent}

Die Abschläge bei Mehrfachabrechnung betragen 10 beziehungsweise $15 \%$. Die Höhe des Abschlages richtet sich nach der entsprechenden EBM-Nummer. Wird bei EBM-Leistungen, die pro Patient begrenzt mehrmals im Quartal durchgeführt und abgerechnet werden dürfen, die Maximalhäufigkeit überschritten, werden zwar alle Leistungen vergütet, allerdings mit einem Abschlag von der Punktzahl in Höhe von 10 \%. Bei Leistungen die pro Patient nur einmal im Quartal abgerechnet werden dürfen, wie zum Beispiel die Grundpauschale, wird bei Abrechnung durch mehrere Ärzte des ASV-Teams ein Abschlag von $15 \%$ vorgenommen.

Dr. Dr. Peter Schlüter 